\title{
Design of Impulse Hydro Turbines
}

\section{John S Anagnostopoulos}

School of Mechanical Engineering, National Technical University of Athens, Greece

Renewable energy sources is the future in electricity and energy production, while the share of hydropower will remain significant, as the developing countries are increasingly exploiting their large hydraulic potential, and the developed world focuses on further production from existing and new sites, even at small and mini scales. Moreover, the use of pumped storage technology to support large RES penetration into electric grids will raise the capacity factor of existing hydropower installations and motivate the construction of new large plants.

The main classical hydraulic turbine types, after their invention in the last centuries, (Francis, 1848; Pelton, 1870; Kaplan, 1913; Turgo, 1919; etc), are still widely used today and will operate for many more years, since they can cover all possible combinations of hydro power site conditions (head, flow rate, and power variation pattern).

The traditional design of these turbines was based on the accumulated experience of the manufacturers and engineers, and from time to time has adopted some improvements, by applying the costly trial-and-error method, and by exploiting rather the expertise and intuition of some engineers than the hydrodynamics theory.

This design practice is changing in the last two decades, and fluid mechanics theory is increasingly applied, thanks to the huge rise of computing power and the available commercial and open-source tools. Today, most large manufacturers make use of modern Computational Fluid Dynamics software in order to analyze and study in detail the flow field development inside the turbines, to test possible design modifications in the computer. Their design sector is staffed by skilled and competent engineers and designers, who have studied numerical modeling and graphical representation method, and are able to use complex computer software or even to develop customized computer algorithms.

Powerful numerical design optimization tools, also developed in recent years, allow the simultaneous variation of numerous design parameters of the turbine (e.g. rotating blades and guide vanes shape, casing and meridian channels geometry), in order to achieve single or multiple objectives (e.g. maximization of energy efficiency and minimization of unwanted mechanisms like cavitation). Multipoint operation and unsteady phenomena, like pressure oscillations, are also modeled and controlled with the aid of specific software, whereas stress analysis tools are combined to improve manufacturing procedures and costs.

The production efficiency of hydro turbines becomes a crucial feature today, not only for the net energy production and economic viability of modern hydropower plants, but also for the competitiveness and market penetration of turbine manufacturers. More and more customers-hydroelectricity producers are increasingly concerned, in addition to the mechanical performance and reliability of the machinery, also about the energy production efficiency of their plants. The latter depends strongly on the hydrodynamic design of hydro turbines and the amount of hydraulic losses during the energy exchange in the machines.

On the other hand, however, small and medium hydro turbine manufacturers, who have not yet established or cannot afford a separate numerical modeling and design department, have started feeling the pressure of modern design practices, and the increasing demands of their customers for provision of detailed operation characteristics of the turbines, along with their electromechanical specifications. Such data can be obtained only by certified and independent testing facilities that are found in a few Universities and research centers, like EPFL in Lausanne, NTNU in Trondheim, and NTUA in Athens.

The flow field in a reaction turbine runner (Francis, Kaplan) can be simulated as steady flow in the rotating system of reference, and in a blade-to-blade periodic section, while time-dependent simulations of the whole domain (including guide vanes and casing) are used separately, mainly to update the boundary conditions of the runner. Consequently, the detailed design variables of the runner can be studied thoroughly, while modern design optimization tools can handle thousands of flow field evaluations in an affordable computer cost, even by a small manufacturer.

The case of impulse turbines is, however, much more difficult, because of the very complex, unsteady, two-phase, free-surface flow, which is developed during the jet-runner interaction [1]. This requires up to two orders of magnitude higher computer processing time per each simulation, than in a reaction turbine runner. Moreover, a number of complex and transient flow mechanisms are involved, like local flow separation and cavitations in the jet impact and bucket cut region, and the back-side flow, which are extremely difficult to be simulated and predicted in a reliable manner. Also, the structure of the jet emerging from the nozzle exhibits certain asymmetries due to the spear valve design and to secondary flows created in the distribution piping system. Jet surface degradation also appears due to interaction with the surrounding air, as also because of the impact of water droplets of runner outflow. Finally, the evacuation of the outflow water plays important role, because after exiting from the buckets, the flow impacts on the inner casing surfaces, thus creating a misty and droplet environment in the casing. Part of this water may re-enter the runner and reduce its hydraulic efficiency, while the misty environment increases the mean air density and hence the ventilation losses of the rotating runner. All the above mechanics cannot be easily and reliably modeled.

Turgo impulse turbines can operate at higher specific speeds, and hence at higher flow rates than Pelton turbines. However, the blades of efficient Turgo runners must have complex 3D shapes and the attained hydraulic efficiency is much more sensitive on their exact design. Our studies have shown that even small shape variations of the blades may lead to large efficiency differences of the order of 5-10 percentage units

*Corresponding author: John S Anagnostopoulos, Associate Professor, Schoo of Mechanical Engineering, National Technical University of Athens, Greece, Tel: +30 210 7721080; E-mail: anagno@fluid.mech.ntua.gr

Received November 06 2013; Accepted November 06, 2013; Published November 15, 2013

Citation: Anagnostopoulos JS (2013) Design of Impulse Hydro Turbines. J App Mech Eng 2: e123. doi:10.4172/2168-9873.1000e123

Copyright: (ㅇ 2013 Anagnostopoulos JS. This is an open-access article distributed under the terms of the Creative Commons Attribution License, which permits unrestricted use, distribution, and reproduction in any medium, provided the original author and source are credited. 
[2]. For this reason, only few manufacturers construct and provide Turgo turbines of hydrodynamic design above a certain size.

Due to the above flow and design complexities, a complete multiparametric design optimization of impulse hydro turbines using conventional Computational Fluid Dynamics software (commercial or in-house), requires huge computing times and large parallel computing systems, that cannot be procured by most manufacturers. The application of meshless particulate methods like the Smoothed Particle Hydrodynamics [3-5] will permit the incorporation of Graphics Processing Units technology for the flow solvers, which seems quite promising towards a significant acceleration of numerical design optimization in impulse hydro turbines.

An alternative methodology is recently developed in our Laboratory of Hydraulic Turbo machinery (http://www.fluid.mech.ntua.gr/lht/) for the simulation and optimum design of impulse turbine runners [6]. The Fast Lagrangian Simulation method (FLS) is based on a Lagrangian approach, in which representative trajectories of flow particles are tracked, after introducing suitable terms in the particle motion equations, in order to account for the various hydraulic losses and the surface flow spreading in the buckets/blades surface. Adjustment of these terms with the aid of experimental data or results from CFD software can lead to very encouraging results concerning the reliability of prediction of flow evolution, and the runner hydrodynamic behavior and hydraulic efficiency. In addition, a complete jet-runner interaction simulation can be performed only in a few CPU seconds in a modern personal computer, compared to several hours needed to solve the full Reynolds Averaged Navier Stokes (RANS) equations.

This tool, combined with powerful stochastic optimization software [7], is being used in our Lab to design improved Pelton and Turgo runners for existing and new small hydropower plants. In parallel, a number of scaled models and of small impulse turbines have been constructed, installed, and tested in the Lab. With the aid of these experimental data the FLS methodology was validated and further tuned $[2,8]$. Also, more sophisticated tools, like RANS and SPH numerical algorithms, are used to improve the design of other turbine parts like the water distribution and injection system [9].

In the near future, it is expected that the numerical design of both reaction and impulse hydro turbines will be incorporated also in small and medium manufacturing enterprises in order to survive in the increasing international competitive market. Improved customized turbines design will become desirable also for small and mini power classes, and will constitute a competitive advantage, if provided at low computer cost. In addition to smooth and reliable operation of the turbines, high energy efficiency will be required, that should be certified by complete hill-charts of machine operation, provided by independent testing facilities. This will motivate further development of specific flow modeling tools and laboratory installations with the right instrumentation and measuring equipment, in order to serve the increasing needs of small hydro-manufacturers. All these efforts will significantly contribute to a better understanding of the complex flow mechanisms that are encountered in impulse hydro turbines, and to maximization of their performance and efficiency.

\section{References}

1. Perrig A (2007) "Hydrodynamics of the free surface flow in Pelton turbine buckets", Ph.D. Thesis, École Polytechnique Fédérale de Lausanne, Switzerland.

2. Anagnostopoulos J, Koukouvinis Ph, Stamatelos F, Papantonis D (2012) Optimal design and experimental validation of a Turgo model hydro turbine. $11^{\text {th }}$ Conference on Engineering Systems Design and Analysis (ESDA), ASME, Nantes, France.

3. Marongiu J Ch, Leboeuf F, Caro J, Parkinson E (2010) "Free surface flows simulations in Pelton turbines using an hybrid SPH-ALE method", Journal of Hydraulic Research 48 (Suppl. 1): 40-49.

4. Koukouvinis PK, Anagnostopoulos J, Papantonis D (2013) An improved MUSCL treatment for the SPH-ALE method: Comparison with the standard $\mathrm{SPH}$ method for the jet impingement case. International Journal for Numerical Methods in Fluids 71: 1152-1177.

5. Koukouvinis PK, Anagnostopoulos J, Papantonis D (2011) SPH method for flow predictions at a Turgo impulse turbine: Comparison with Fluent. World Academy of Science, Engineering and Technology 79: 659-666.

6. Anagnostopoulos J, Papantonis D (2012) A fast Lagrangian simulation method for flow analysis and runner design in Pelton turbines. J Hydrodynam 24: 930 941.

7. Karakasis M, Giannakoglou KC (2006) On the use of metamodel-assisted multi-objective evolutionary algorithms. Eng Optimiz 38: 941-957.

8. Stamatelos F, Anagnostopoulos J, Papantonis D (2011) Performance measurements on a Pelton turbine model. J Power and Energy 225: 351-362.

9. Koukouvinis PK, Anagnostopoulos J, Papantonis D (2009) Flow modelling in the injector of a Pelton turbine, $4^{\text {th }}$ International SPHERIC Workshop, Nantes, France. 